\title{
The impact of breathing motion versus heterogeneity effects in lung cancer treatment planning
}

\author{
Mihaela Rosu \\ Department of Radiation Oncology, The University of Michigan, Ann Arbor, Michigan 48109-0010 \\ Indrin J. Chetty \\ Department of Radiation Oncology, University of Nebraska Medical Center, Omaha, Nebraska 68198-7521 \\ Daniel S. Tatro and Randall K. Ten Haken \\ Department of Radiation Oncology, The University of Michigan, Ann Arbor, Michigan 48109-0010
}

(Received 4 December 2006; revised 30 January 2007; accepted for publication 13 February 2007; published 23 March 2007)

\begin{abstract}
The purpose of this study is to investigate the effects of tissue heterogeneity and breathing-induced motion/deformation on conformal treatment planning for pulmonary tumors and to compare the magnitude and the clinical importance of changes induced by these effects. Treatment planning scans were acquired at normal exhale/inhale breathing states for fifteen patients. The internal target volume (ITV) was defined as the union of exhale and inhale gross tumor volumes uniformly expanded by $5 \mathrm{~mm}$. Anterior/posterior opposed beams (AP/PA) and three-dimensional (3D)conformal plans were designed using the unit-density exhale ("static") dataset. These plans were further used to calculate (a) density-corrected ("heterogeneous") static dose and (b) heterogeneous cumulative dose, including breathing deformations. The DPM Monte Carlo code was used for dose computations. For larger than coin-sized tumors, relative to unit-density plans, tumor and lung doses increased in the heterogeneity-corrected plans. In comparing cumulative and static plans, larger normal tissue complication probability changes were observed for tumors with larger motion amplitudes and uncompensated breathing-induced hot/cold spots in lung. Accounting for tissue heterogeneity resulted in average increases of $9 \%$ and $7 \%$ in mean lung dose (MLD) for the $6 \mathrm{MV}$ and $15 \mathrm{MV}$ photon beams, respectively. Breathing-induced effects resulted in approximately $1 \%$ and 2\% average decreases in MLD from the static value, for the 6 and 15 MV photon beams, respectively. The magnitude of these effects was not found to correlate with the treatment plan technique, i.e., AP/PA versus 3D-CRT. Given a properly designed ITV, tissue heterogeneity effects are likely to have a larger clinical significance on tumor and normal lung treatment evaluation metrics than four-dimensional respiratory-induced changes. (c) 2007 American Association of Physicists in Medicine.
\end{abstract}

[DOI: $10.1118 / 1.2713427$ ]

Key words: lung heterogeneity, 4D planning, cumulative doses, Monte Carlo

\section{INTRODUCTION}

Radiation therapy is one of the principal treatment options for medically inoperable lung tumors. However, even with significant advances in the delivery of radiation, 5-y survival rates remain poor for patients with advanced stage disease. ${ }^{1-5}$ As such, research efforts have been devoted toward individualizing the radiation therapy process to each patient's specific anatomy and physiology in the planning and the delivery of high dose conformal therapy. These efforts have been encouraged by studies that indicate that higher doses can be safely delivered to limited lung volumes, ${ }^{6-14}$ as well as the fact that higher target doses relate to improved tumor control. ${ }^{15-18}$ It should, however, be kept in mind that these results may carry some bias and limitations. This is attributed to the fact that the doses quoted in trials are usually calculated using no or limited heterogeneity correction algorithms. Additionally, changes in dose due to organ motion and deformation are not generally accounted for in dose evaluation. As we move toward individualization in the treatment planning process using advanced methods, such as fourdimensional (4D) imaging, it becomes critical that we incorporate methods to correctly accumulate these doses using the patient CT-based densities, ultimately to more accurately assess the dose actually delivered to the patient.

To better estimate doses in anatomies with heterogeneous tissue densities, such as the lung, accurate dose calculation engines have been developed. In this regard, Monte Carlo based dose calculation algorithms are believed to be the most accurate. ${ }^{19-29}$ They can provide dose calculations that properly account for patient-specific physical descriptions of anatomy (including electron density and atomic number information), and for the charged particle transport processes that are influenced by different tissue properties. However, although many studies have demonstrated that significant errors are present when homogeneous media is assumed where heterogeneities are actually present [see AAPM Report No. 
TABLE I. Patient information regarding tumor location, size, motion amplitude, and prescription doses for all treatment plans $(\mathrm{UR}=$ upper right lobe, $\mathrm{UL}=$ upper left lobe, $\mathrm{MR}=$ medial right, $\mathrm{LR}=$ lower right lobe, $\mathrm{LL}$ $=$ lower left lobe). The breathing motion amplitude was derived from the center-of-mass of the tumor displacement between exhale and inhale.

\begin{tabular}{|c|c|c|c|c|c|c|}
\hline \multirow[b]{3}{*}{ Patient } & \multirow{3}{*}{$\begin{array}{l}\text { Tumor } \\
\text { location }\end{array}$} & \multirow{3}{*}{$\begin{array}{l}\text { GTV } \\
\text { size } \\
\left(\mathrm{cm}^{3}\right)\end{array}$} & \multirow{3}{*}{$\begin{array}{l}\text { Breathing } \\
\text { motion } \\
\text { amplitude } \\
(\mathrm{cm})\end{array}$} & \multicolumn{3}{|c|}{ Prescription dose (Gy) } \\
\hline & & & & \multicolumn{2}{|c|}{$6 \mathrm{MV}$} & \multirow{2}{*}{$\begin{array}{c}15 \mathrm{MV} \\
\begin{array}{c}3 \mathrm{D} \\
\text { conforma }\end{array}\end{array}$} \\
\hline & & & & $\mathrm{AP} / \mathrm{PA}$ & conformal & \\
\hline 1 & MR & 67 & 1.2 & 42 & 84 & 84 \\
\hline 2 & UR & 300 & 0.9 & 48 & 72 & 76 \\
\hline 3 & LR & 23 & 1.1 & 100 & 100 & 100 \\
\hline 4 & LR & 160 & 1.7 & 100 & 100 & 100 \\
\hline 5 & UR & 816 & 0.1 & 48 & 60 & - \\
\hline 6 & UR & 103 & 0.5 & 50 & 52 & - \\
\hline 7 & UR & 29 & 0.6 & 100 & 100 & - \\
\hline 8 & UL & 5 & 0.1 & 100 & 100 & - \\
\hline 9 & UL & 82 & 1.0 & 48 & 74 & - \\
\hline 10 & ML & 560 & 0.7 & 66 & 60 & - \\
\hline 11 & MR & 17 & 0.6 & 100 & 100 & - \\
\hline 12 & UL & 89 & 1.6 & 100 & 100 & - \\
\hline 13 & LR & 490 & 0.8 & 46 & 100 & - \\
\hline 14 & MR & 192 & 2.1 & 54 & 70 & - \\
\hline 15 & UR & 50 & 1.4 & 48 & 100 & - \\
\hline
\end{tabular}

85 (Ref. 30) for references, reviews, and summary], it is still debated whether or not heterogeneity corrections should be used. $^{31-37}$

Breathing motions and the distortions associated with them triggered the development of sophisticated CT systems that allow the acquisition of time-dependent images that extend across the entire breathing cycle, setting the basis for what is nowadays termed as $4 \mathrm{D}$ radiation therapy. ${ }^{38}$ Of course, much more work is warranted before many of the uncertainties introduced by the additional components involved in a 4D therapy process (e.g., image registration, data sorting, inter- and intra-fraction breathing variability, to name a few) are fully understood and quantified. Even if a $4 \mathrm{D}$ radiotherapy treatment could be assumed to be error free, the benefits of using a technology that demands such large resource utilization are yet to be proven, and the classes of patients that could make best use of these advances are yet to be identified.

The present study compares the dosimetric effects of heterogeneous media versus breathing-induced motion and deformation for patients with lung tumors treated with conformal radiotherapy. The comparison was performed by assessing the impact of heterogeneities and breathing motion on clinically relevant target and normal tissue treatment evaluators such as equivalent uniform dose (EUD), normal tissue complication probability (NTCP), and mean lung dose (MLD). A secondary goal of the study was to identify scenarios were heterogeneities or respiratory induced motion were more likely to have a clinically important effect, with emphasis on the analysis of motion/deformation effects, an area that has not been extensively investigated in the literature.

\section{METHODS AND MATERIALS}

\section{A. Patient data}

Fifteen patients with unresectable lung tumors were involved in this study under a protocol approved by the Internal Review Board at the University of Michigan Health Systems. These patients had various tumor sizes, locations, and motion amplitudes (Table I). Exhale and inhale scans acquired at normal breath-hold during the same session were available for all patients.

\section{B. Treatment planning}

For each patient, the exhale and inhale gross tumor volumes (GTVs) were contoured by a physician, and uniformly expanded $0.5 \mathrm{~cm}$ to form clinical tumor volumes (CTVs). An internal target volume (ITV) was then defined as the union of the exhale and inhale CTVs. In this study we assumed that (a) the setup errors were dealt with prior to radiation delivery and (b) the breathing motion can be described by the assumed model, ${ }^{39}$ with no inter- or intra-fraction variations. That is, all other factors that would normally lead to further expansion of the ITV to form a planning target volume were not considered here in order to isolate the breathing motion and tissue density effects, and their relative magnitudes. Critical organs (spinal cord, esophagus, heart, lungs) were also contoured within the treatment planning system.

Each patient was planned with an anterior/posterior opposed beams plan (AP/PA) plan and with a threedimensional (3D)-conformal plan (multiple coplanar beams and segments) using $6 \mathrm{MV}$ photon beams. The intent here was to investigate whether the heterogeneity and motion ef- 
fects correlate with the dose distribution conformality. The plans were designed on the exhale (static, planning) dataset, assuming unit density. For four patients that had larger motion amplitudes 3D-conformal plans using $15 \mathrm{MV}$ photon beams were designed as well, again using the exhale dataset and assuming unit density. The number of beams and segments for the 3D-conformal plans ranged between 5 and 11 . The prescription doses for each scenario are summarized in Table I.

The dose distributions were calculated using the DPM Monte Carlo code ${ }^{26}$ which has been integrated within the UMPlan in-house treatment planning system. ${ }^{40}$ The electron step size was $2 \mathrm{~mm}$, and the low energy electron and photon cutoff kinetic energies were 200 and $50 \mathrm{keV}$, respectively. $1.5 \times 10^{9}$ histories were simulated per plan, resulting in $1-\sigma$ statistics of better than $0.5 \%$ on average within the target. ${ }^{41}$ The plans calculated using the exhale homogeneous dataset were normalized to $100 \%$ at the isocenter. All the other plans were normalized to the isocenter dose from the reference exhale homogeneous dose distribution to illustrate dose changes for the same number of monitor units per beam as in the reference exhale plan. The dose grid size in all dimensions was $3.5 \mathrm{~mm} .^{42}$

The plans were optimized to cover the ITV with $100 \pm 5 \%$ of the assigned prescription dose (Table I) while keeping the critical normal tissues below tolerances: ${ }^{6,13,18}$ the spinal cord must remain below a dose equivalent to $50 \mathrm{~Gy}$ in 2 Gy fractions; the esophagus effective volume $\left(V_{\text {eff }}\right)$ (Ref. 43) computed with a normalization dose equivalent to $72 \mathrm{~Gy}$ in 2 Gy fractions must be less than $1 / 3$ of the organ volume; the heart $V_{\text {eff }}$ computed with normalization doses equivalent to $40 \mathrm{~Gy}$ and $65 \mathrm{~Gy}$ in $2 \mathrm{~Gy}$ fractions must be less than 1 and $1 / 3$ of the organ volume, respectively; the normal lung $V_{\text {eff }}$ normalized to the prescription dose must be less than 0.4 , and is used to determine the assigned dose $\mathrm{e}^{12,13,18}$ to be consistent with the $15 \%$ target iso-NTCP of grade 3 or higher radiation pneumonitis model ${ }^{43,44}$ (model parameters were ${ }^{45} \mathrm{TD}(50)$ $=30.8 \mathrm{~Gy}, m=0.99, n=0.37)$. If any of these tolerance doses could not be met, the dose was adjusted until the critical structure dose was not exceeded.

Each plan designed on the exhale homogeneous dataset (beam weights and monitor units) was also used for the calculation of the heterogeneous dose distribution. For evaluating the inhomogeneity effects on tumors and normal lung doses for each patient, the homogeneous and the heterogeneous exhale dose distributions were compared (Scenario 1).

Each reference static exhale plan (beam weights and monitor units) was also used for heterogeneity-corrected dose computation on the inhale dataset. The inhale dose distribution was then mapped back onto the exhale dataset using the transformation provided by a deformable B-spline registration technique. ${ }^{46}$ A time-weighted summation of the exhale and inhale doses was used to estimate the cumulative heterogeneous dose that would be received by a patient during free breathing radiation delivery. ${ }^{42,47}$ The weighting coefficients used were $70 \%$ for exhale and $30 \%$ for inhale, as derived from a breathing probability function assumed for all patients for planning. ${ }^{39}$ The comparison between the cumulative and exhale heterogeneous dose distributions (Scenario 2) provided information regarding the breathing-induced motion effects. We also assessed the cumulative effect of heterogeneity and motion effects by comparing the heterogeneous cumulative and the homogeneous static dose distributions (Scenario 3). All three scenarios are depicted in Fig. 1.

\section{Heterogeneity and breathing-induced motion and deformation assessment}

The effects of heterogeneity and/or breathing-induced motion and deformation were assessed qualitatively, through dose difference displays, and quantitatively, thorough treatment metrics commonly used in lung cancer treatment planning: CTV EUD, ${ }^{48}$ MLD, and NTCP. These metrics were evaluated for the anatomical structures segmented on the exhale dataset. The changes in isocenter doses from the exhale homogeneous values were also investigated. The clinical significance of the changes induced by inhomogeneities and/or motion was determined based on previous work at our institution: ${ }^{49,50}$ for the tumor, the change in tumor dose is clinically important if it equals or exceeds a treatment fraction size (e.g., $2 \mathrm{~Gy}$ ); for the normal lung tissue, the change in lung NTCP for a given dose distribution from a reference dose distribution is clinically important if a change in the prescription dose of at least one treatment fraction is needed in order to achieve the same complication probability level from the reference dose distribution (iso-NTCP based dose prescription).

\section{RESULTS}

\section{A. Image registration assessment}

Registration is an important step in the computation of cumulative doses from multiple datasets. The accuracy of the process is usually described by the accuracy of bringing into alignment anatomical landmarks and was found to be about $2-3 \mathrm{~mm}$, on average, for the patient population involved in this study. The visual inspection of the overlapped exhale and the deformed inhale datasets (inhale CT scans distorted to the exhale state) was also used to validate the registration quality and was found to be acceptable for all cases (one example is provided in Fig. 2).

\section{B. Heterogeneity effects}

Shown in Fig. 3 are several dose difference displays between heterogeneous and homogeneous dose distributions. In general, the heterogeneous dose calculation predicts higher doses than the homogeneous dose calculation for both tumors and lungs, as indicated by the hot spots. However, cold spots arise near interfaces and at the beam edges. The heterogeneity effects on CTV EUD, and the prescription dose changes required in the heterogeneous dose calculation to restore the lung NTCP from the homogeneous calculation are illustrated in Figs. 4 and 5 (dark-gray bars). 

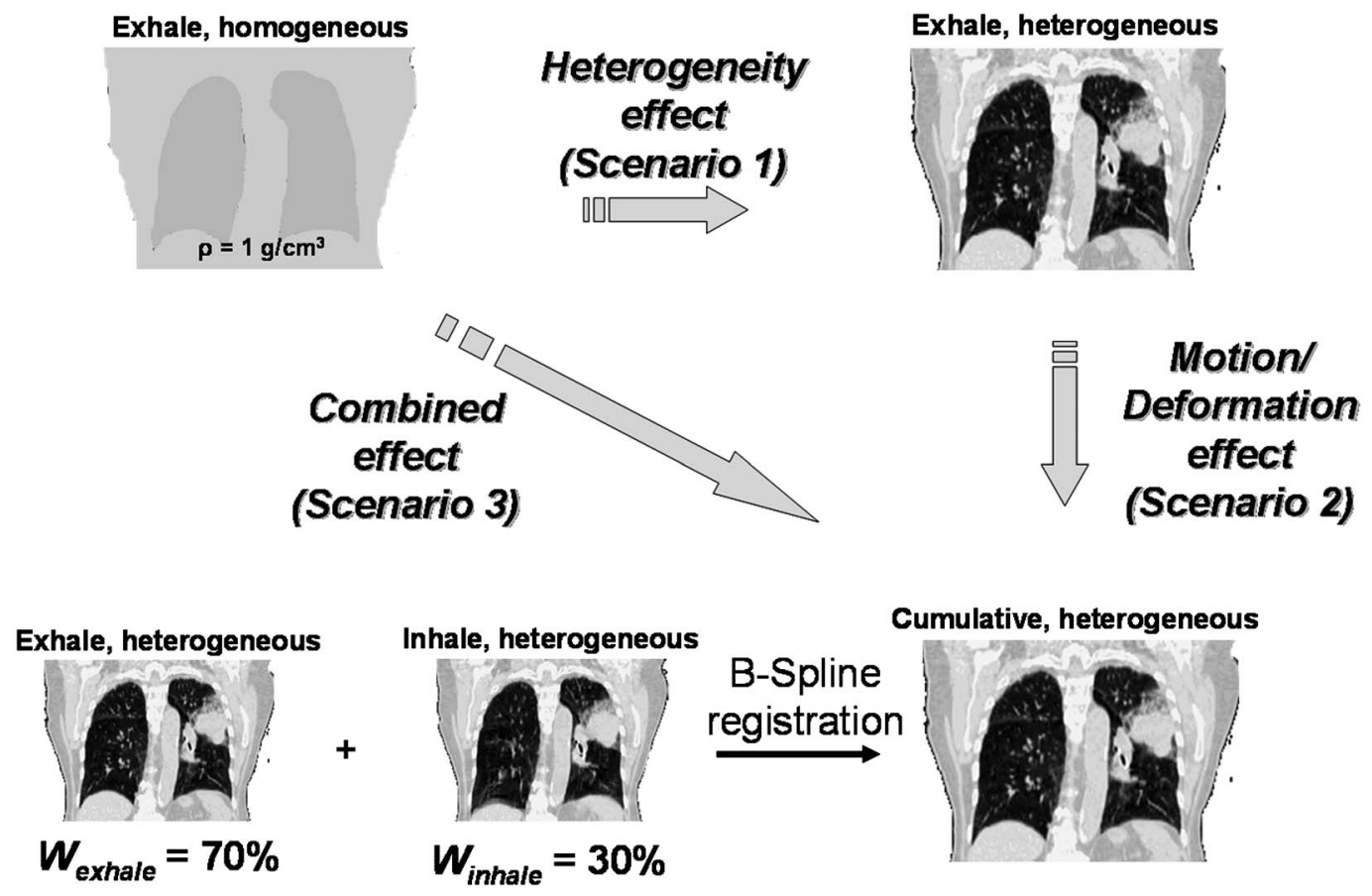

FIG. 1. A treatment plan is designed on the exhale dataset, assuming homogeneous media $\left(\rho=1 \mathrm{~g} / \mathrm{cm}^{3}\right)$. The plan is also used for heterogeneous dose calculation on the exhale and inhale datasets. The heterogeneous inhale dose distribution is scored back onto the exhale dataset and the time weighted sum of the exhale (70\%) and inhale (30\%) doses estimates the cumulative dose to be received by a patient during free breathing delivery of the radiation treatment. The comparison between exhale homogeneous and heterogeneous doses illustrates the inhomogeneity effects (Scenario 1). The comparison between exhale and cumulative heterogeneous dose illustrates the effects induced by breathing-induced motion and deformation (Scenario 2). The comparison between the exhale homogeneous and cumulative heterogeneous doses illustrates the combined effect of motion and inhomogeneities.

For tumors, the largest increases in the heterogeneous EUD (over 8 Gy) were found for Patient 3 [6 MV, AP/PA plan; Fig. 3(a)] and Patient 1 [6 MV, conformal plan; Fig. 3(d)], whereas the smallest increase was observed for Patient 5 [6 MV, AP/PA plan; Fig. 3(b)]. Decreases of about 2 Gy in the heterogeneous EUD were observed for Patient $8[6 \mathrm{MV}$, conformal plan; Fig. 3(e)] and Patient 3 [15 MV, conformal plan; Fig. 3(f)].

In the heterogeneous calculation, the correction factors for the isocenter doses (i.e., the ratio of isocenter doses in the

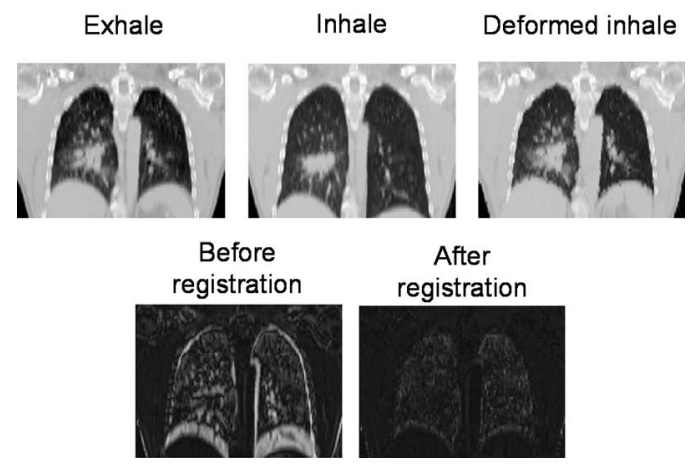

FIG. 2. B-spline deformable registration between the exhale and inhale datasets for Patient 14 for an example coronal cut. All cuts (exhale, inhale, deformed inhale) are displayed for the same plane in the treatment room coordinate system. The bottom row illustrates the intensity difference map between exhale and inhale images (before registration), as well as between exhale and deformed inhale images (after registration); the light-colored regions indicate misalignments. heterogeneous versus the homogeneous plans), averaged 1.046 (range: 1-1.16) for the AP/PA 6-MV plans, 1.035 (range: $0.996-1.11$ ) for the conformal $6 \mathrm{MV}$ plans, and 1.042 (range: $1.02-1.08$ ) for the conformal $15 \mathrm{MV}$ plans.

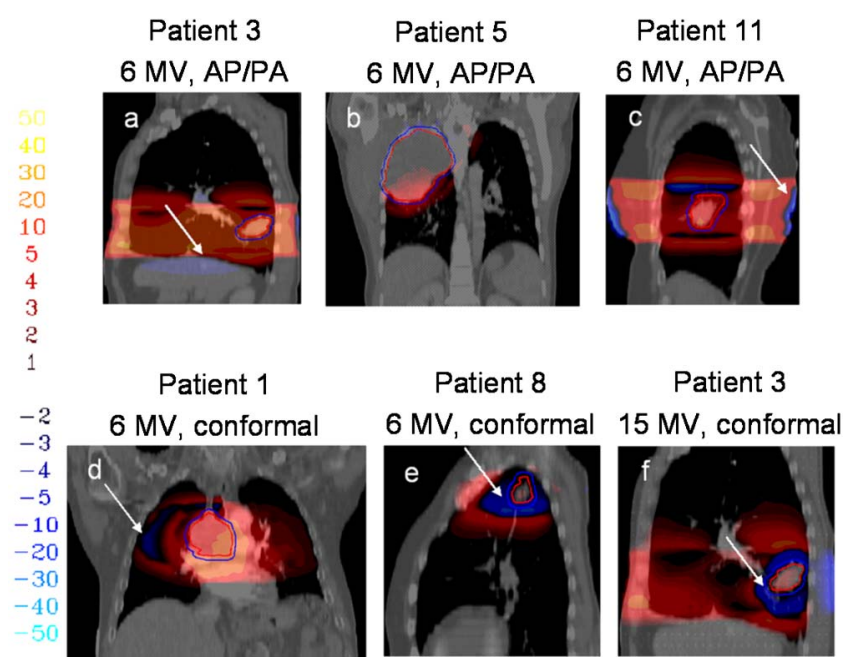

FIG. 3. Dose difference displays between heterogeneous and homogeneous dose distributions computed on the exhale dataset for several patients. The hot/cold spots indicate regions were the heterogeneous dose distribution predicts larger/smaller values than the homogeneous dose distribution. The arrows indicate cold spots near interfaces in the heterogeneous dose calculation. The numbers indicate percent dose differences relative to the prescription dose. 

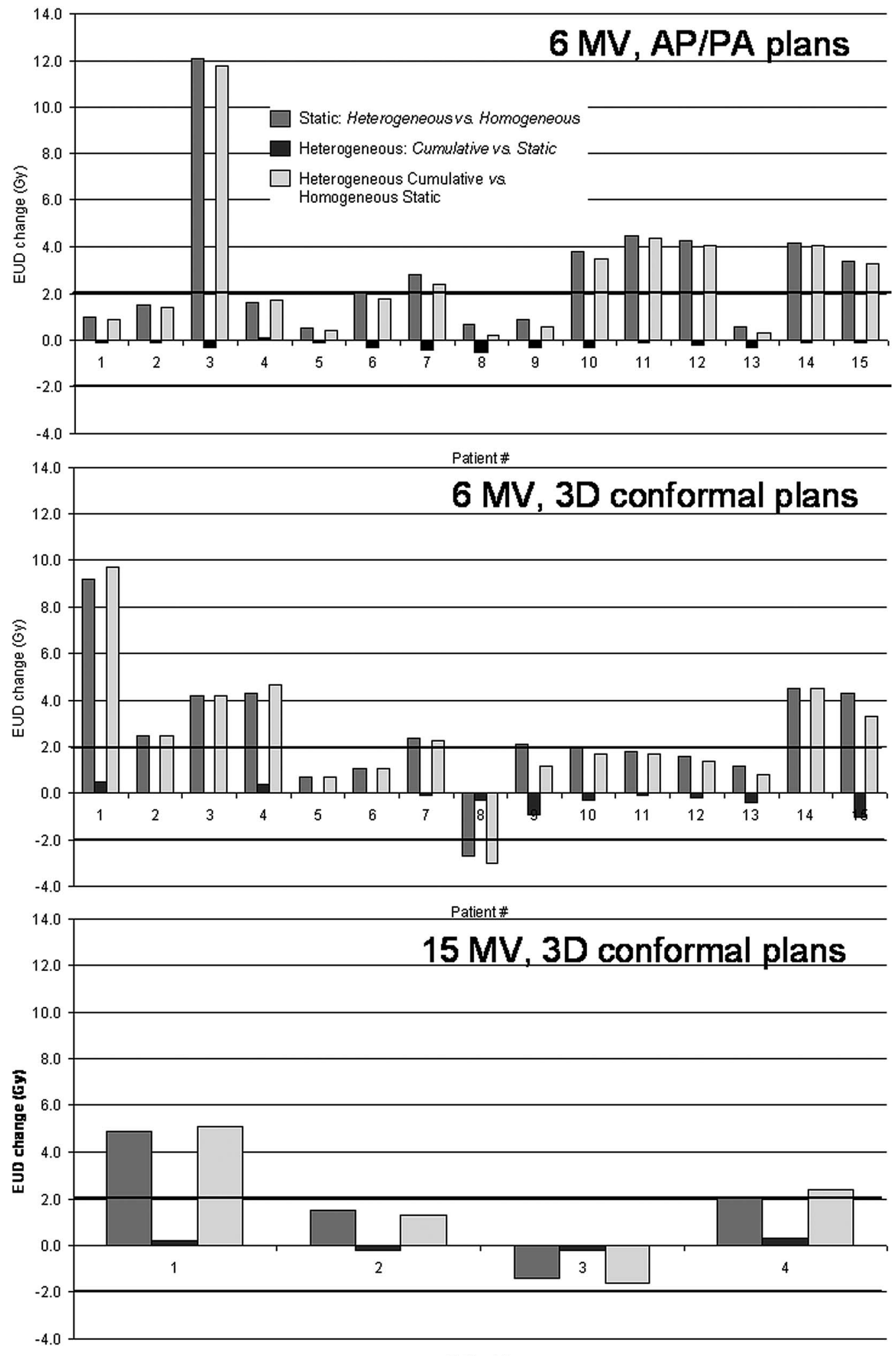

Patient \#

FIG. 4. Tumor EUD (Gy) changes from their homogeneous exhale values as a result of heterogeneity (dark gray bars), motion/deformation (black bars), and the combined effect of motion and heterogeneity (light gray bars). The dark horizontal lines ( $\pm 2 \mathrm{~Gy}$ ) correspond to the treatment fraction size assumed for the patients from this study. 

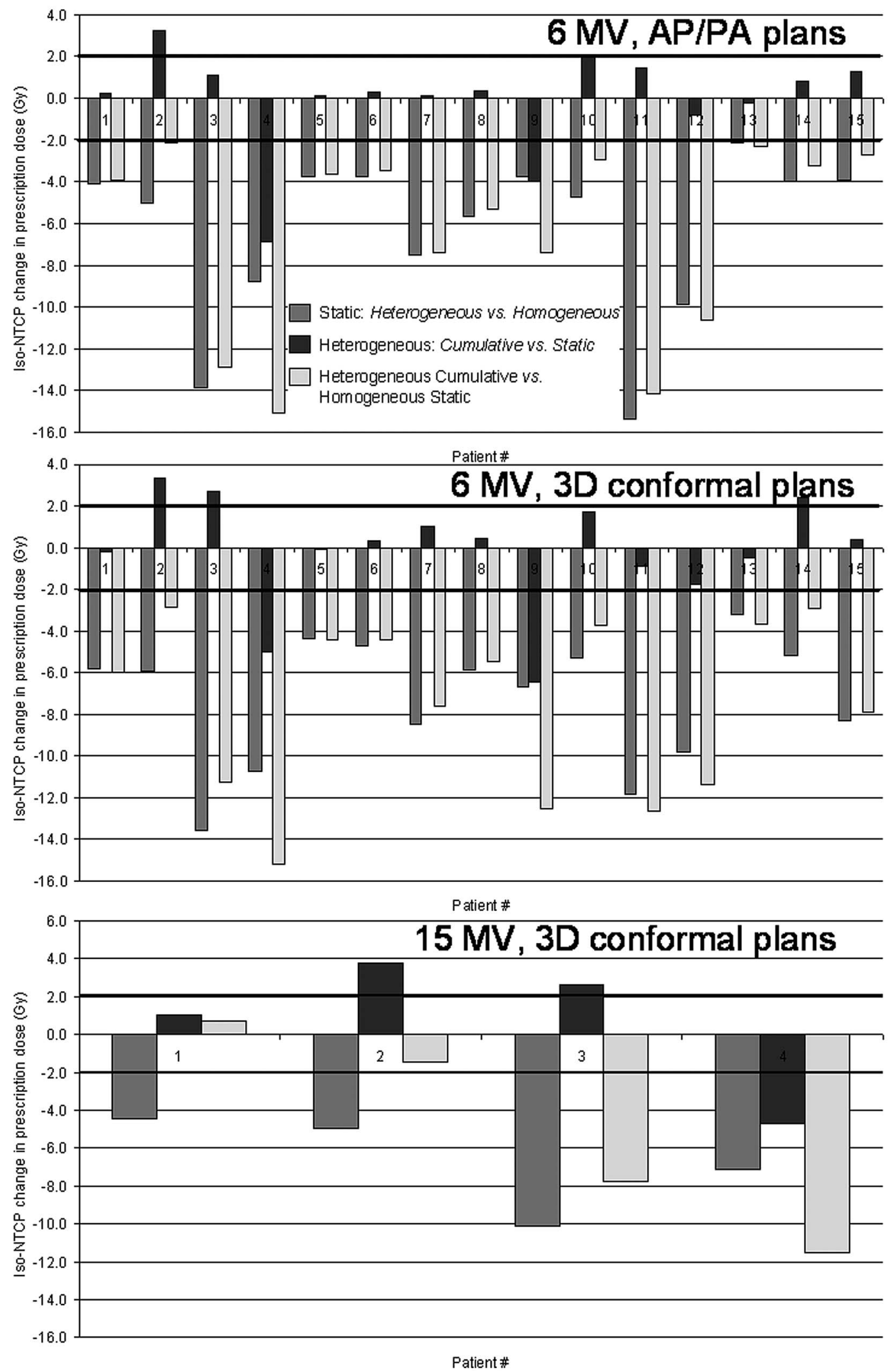

FIG. 5. Changes in the prescription dose from the homogeneous exhale value required in an iso-NTCP protocol when heterogeneities (dark gray bars), motion/deformation (black bars), and the combined effect of motion and heterogeneity (light gray bars) are included in dose calculations. The dark horizontal lines $( \pm 2 \mathrm{~Gy})$ correspond to the treatment fraction size assumed for the patients from this study. 
With regard to the normal lung tissue, in all cases the heterogeneous calculations predicted larger doses than the corresponding homogeneous calculations. The decrease in the prescription dose that would be required in the heterogeneous dose calculation in order to achieve the complication probability level estimated in the homogeneous dose calculation (illustrated in Fig. 5) was at least one treatment fraction in all cases. The largest changes were $\sim 16$ Gy $(8$ fractions) for Patient 11 [6 MV, AP/PA plan; Fig. 3(c)] and $\sim 14$ Gy (7 fractions) for Patient 3 [6 MV, AP/PA and conformal plans; Figs. 3(a) and 3(f)].

Shown in Fig. 6 are scatter plots of the homogeneous versus the heterogeneous MLDs for the $6 \mathrm{MV}$ and the $15 \mathrm{MV}$ beams. For the patients involved in this study, the average change in the heterogeneous MLD from the homogeneous value, as predicted by the linear fit through origin from Fig. 6, indicates an increase of 9\% for the $6 \mathrm{MV}$ photons (range: $5 \%-16 \%$ ), and $7 \%$ for the $15 \mathrm{MV}$ beam (range: $6 \%-11 \%$ ). The $6 \mathrm{MV}$ data from the plot include the AP/PA and the conformal plan MLDs. The average scaling factors between the heterogeneous and the homogeneous MLDs was not shown to correlate with the dose distribution conformality.

\section{Breathing-induced motion and deformation effects}

Figure 7 displays dose difference maps between heterogeneous cumulative and heterogeneous static dose distributions. Breathing redistributes doses, creating hot and cold spots in the cumulative dose. The motion-induced effects on tumor EUD, and the prescription dose changes required in the cumulative dose distribution to restore the static lung NTCP are illustrated in Figs. 4 and 5 (black bars).

A slight decrease in tumor coverage was observed in most cases, except for Patient 4 (all plans) and Patient 1 (6 and $15 \mathrm{MV}$ conformal plans). The isocenter doses for the cumulative distributions were virtually unchanged from their static values.

The normal tissue doses increased, decreased, or remained unchanged, depending on the breathing amplitude and the tumor location. The comparison between static and cumulative normal lung doses indicates that changes in the prescription dose in an iso-NTCP based dose escalation protocol is required in some cases. The largest changes relative to the static dose distributions were observed as follows: $\sim 6$ Gy (3 fractions) decrease for Patient 4 (6 MV, AP/PA plan) and Patient 9 (6 MV, conformal plan); $\sim 4$ Gy (2 fractions) increase for Patient 2 [15 MV, conformal plan; Fig. 7(b)]; $\sim 4$ Gy (2 fractions) decrease for Patient 4 [6 MV, conformal plan; Fig. 7(d) and $15 \mathrm{MV}$, conformal plan], and Patient 9 [6 MV, AP/PA plan; Fig. 7(f)]. For the patients involved in this study, the average change in the cumulative heterogeneous dose MLD relative to the static heterogeneous dose, as predicted by the linear fit through the origin shown in Fig. 6 , indicates $\sim 1 \%$ decrease for the $6 \mathrm{MV}$ beam (range: $-4 \%-10 \%$ ) and $\sim 2 \%$ decrease for the $15 \mathrm{MV}$ beam (range: $-5-5 \%$ ). The $6 \mathrm{MV}$ data from the plot include the AP/PA and the conformal plan MLDs. The average scaling factors between the cumulative and the static MLDs were found not to correlate with the dose distribution conformality.

\section{Heterogeneity versus breathing-induced motion and deformation effects}

The combined effect of heterogeneity and breathinginduced motion on tumor and normal lung doses, compared with a scenario in which neither one of these effects is accounted for, is illustrated in Figs. 4 and 5 (light gray bars). It can be noticed that heterogeneity effects have the largest contribution toward an accurate estimation of the target coverage in all cases investigated here. For some patients (Patients 2, 4, 9, 10, and 14), however, large breathing-induced excursions resulted in hot or cold spots that were not necessarily simultaneously present in the normal lung tissue. The lack of this compensatory effect between hot and cold spots posed a more significant effect on volume effect indices for these patients.

The magnitude of the changes in dose (as a result of motion or heterogeneity) was not a representative indicator of the effects of motion on lung treatment evaluators. For Patient 14 (Fig. 8), for example, the magnitude of the dosimetric differences induced by the respiratory motion is more than twice the magnitude of the differences induced by heterogeneity, although the impact on MLD/NTCP clearly favors the heterogeneity effects. With the exception of Patient 9 (Fig. 8), the effects of motion on lung treatment metrics, even when significant, were usually smaller than the heterogeneity induced effects.

The isocenter dose changes in the heterogeneous cumulative distributions from the homogeneous static values were the same as those indicated by the heterogeneous static calculation. The corresponding correlation coefficients between MLDs (8\% and 5\% for 6 and 15 MV beams, respectively) were consistent with the cumulative value of the changes generated independently by heterogeneity and motion.

\section{DISCUSSION}

\section{A. Heterogeneity effects}

The increased doses predicted by the heterogeneous dose calculations are a consequence of the reduced attenuation of the primary beam in lung tissue. The effect is generally more pronounced as the amount of lung tissue traversed by the beam becomes larger. For the cases represented in Figs. 3(a) and 3(d) that had the largest increase in the heterogeneous CTV EUD, the ratios between the physical and the radiological path lengths were, on average over all beams, 2.1 and 1.3, respectively. On the other hand, for the case from Fig. 3(b) where the increase in EUD was minimal, the physical and radiological path lengths were almost equal. The physical to radiological path length ratios were fairly large for cases from Figs. 3(e) and 3(f) as well (1.3 and 1.2, respectively). However, a decrease in EUD was found in the heterogeneous calculations for these coin-sized lesions because the range of the electrons in the lung tissue becomes larger 

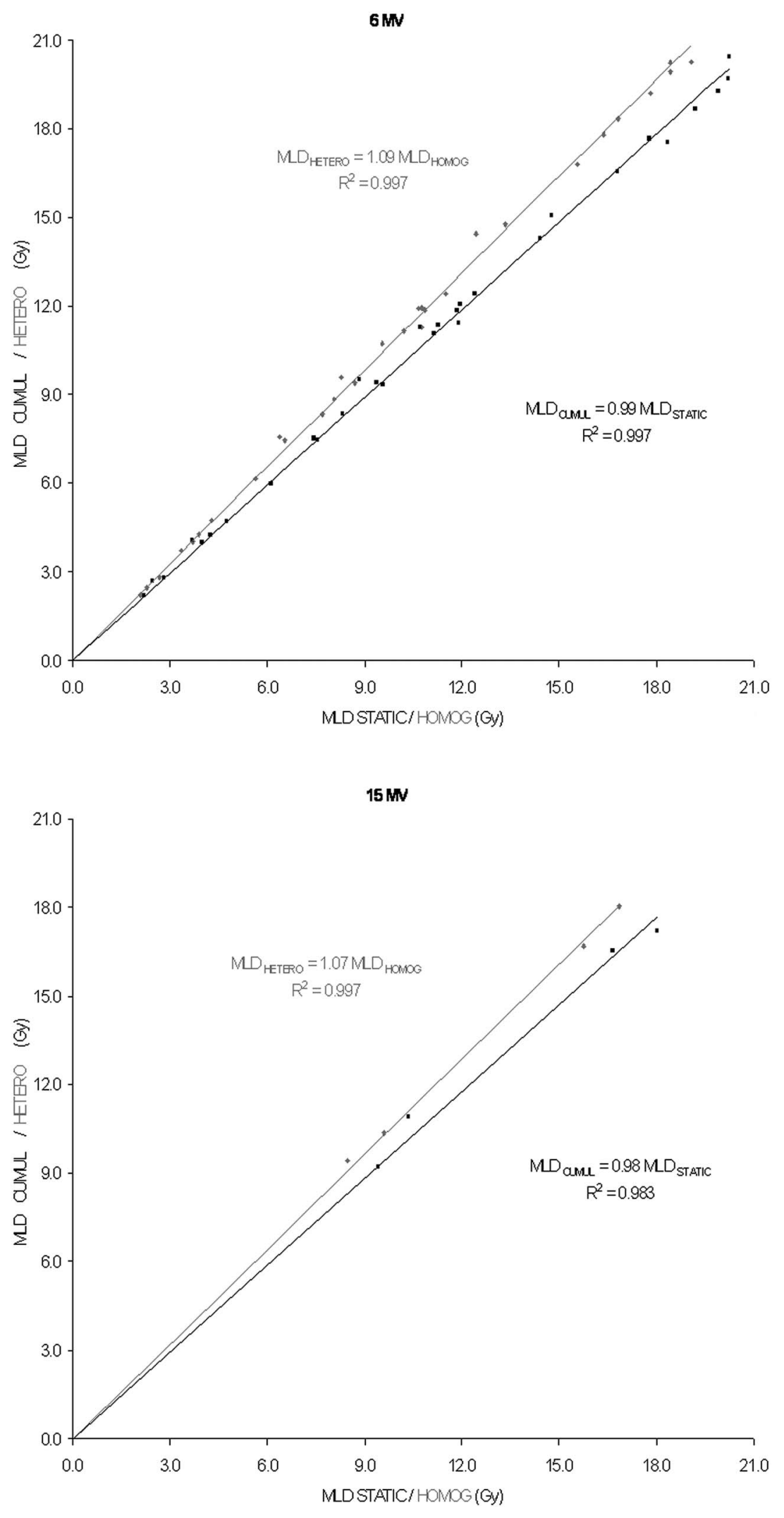

FIG. 6. Scattered plots illustrating the correlations between homogeneous/ cumulative and heterogeneous/static dose distributions for the $6 \mathrm{MV}$ and 15 MV beam energies. than the field size resulting in a loss of charged particle equilibrium. $^{51,52}$ Indeed, Patient 8 [Fig. 3(e)] had a $5 \mathrm{~cm}^{3}$ GTV volume, corresponding to a radius of about $1 \mathrm{~cm}$, comparable with the $\sim 1 \mathrm{~cm}$ electron range in water for a $6 \mathrm{MV}$ photon beam, whereas for Patient 3 [Fig. 3(f)] the GTV volume was $23 \mathrm{~cm}^{3}$, corresponding to a radius of about $1.8 \mathrm{~cm}$, smaller than the $\sim 2.5 \mathrm{~cm}$ electron range in water for a $15 \mathrm{MV}$ photon beam. 


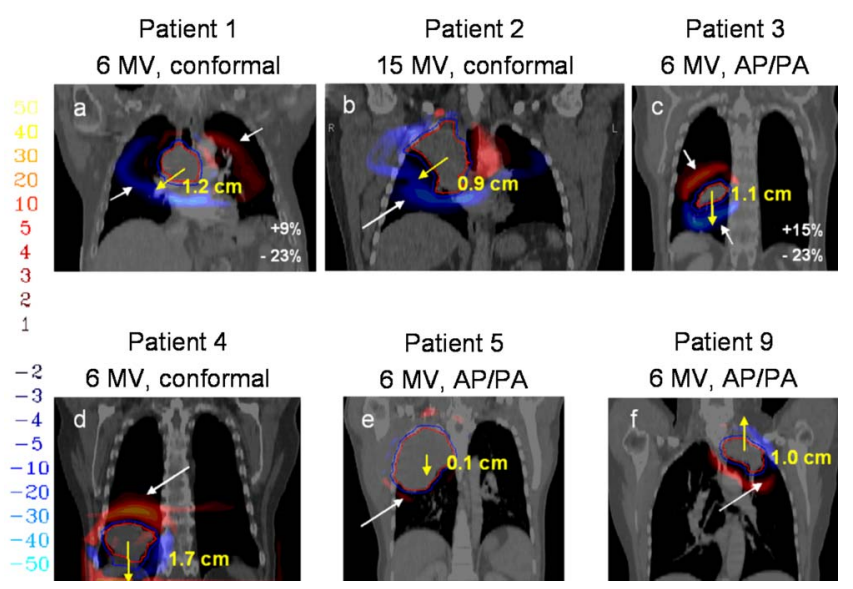

FIG. 7. Dose difference displays between cumulative and static (exhale) dose distributions reported on the exhale dataset for several patients. The color code is the same as in Fig. 3. The white arrows indicate hot and cold spots created by motion and deformation. In addition, the motion amplitude $(\mathrm{cm})$ and the direction along which the tumor has the largest component are indicated. Also shown are maximum and/or minimum point dose differences inside the normal lung tissue. The numbers indicate percent dose differences relative to the prescription dose

The heterogeneities' effects on target doses are different even when prescription doses are similar. Patients 3 and 7 , for example, had similar target volumes (23 and $29 \mathrm{~cm}^{3}$, respectively), and both were prescribed $100 \mathrm{~Gy}$ in the homogeneous AP/PA plans. The heterogeneous EUD, however, was about 8 Gy larger for Patient 3-a consequence of the larger beam path length through lung in the case of Patient 3 (2.1 versus 1.3 average physical to radiological path length ratios).

On average, the heterogeneity increased the isocenter dose. The magnitude of the effect is somewhat different for different beam energies and beam arrangements, with a trend

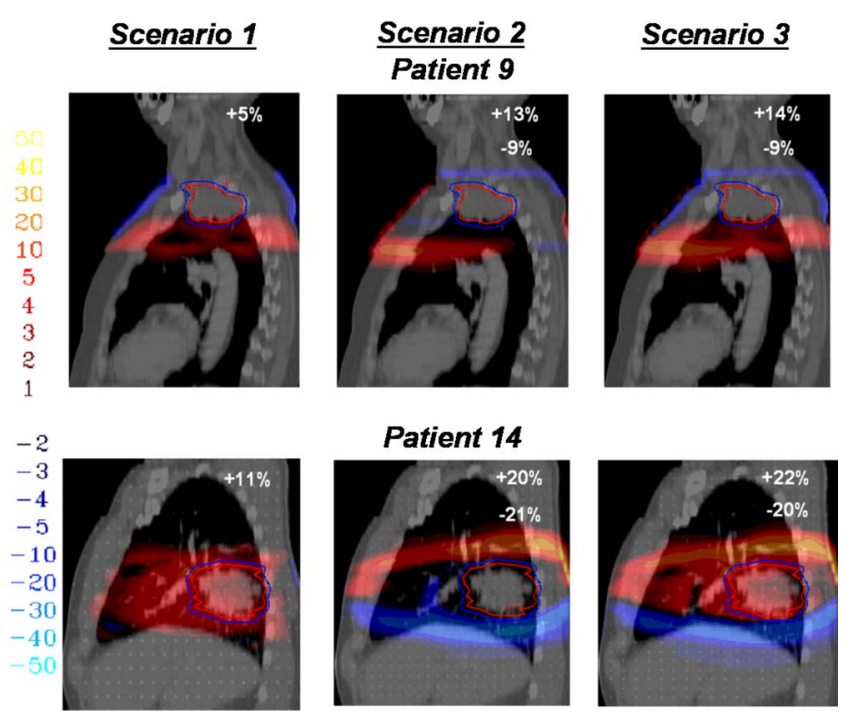

FIG. 8. Difference maps between dose distributions corresponding to the three scenarios investigated in this study, for example, axial, coronal, and sagittal cuts, for Patients 9 and 14. The color code is the same as in Fig. 3. The numbers indicate the maximum and/or minimum point dose differences inside the normal lung tissue. of diminishing effects with increasing beam energy and when multiple beams are used, as indicated by the range of values recorded. Occasionally, a slight decrease in the isocenter heterogeneous dose was observed, owing to larger beam path lengths through bony structures (sternum and vertebral bodies). The results presented in this study are expected and are similar to those previously reported by others. $^{31,32,34}$ The values reported here are, however, slightly larger mainly due to patient selection. For example, if more patients with mediastinal tumors were included, the changes in isocenter dose would be reduced especially for the AP/PA beam technique.

The effect of heterogeneities on the prediction of the radiation-induced pneumonitis is still controversial. Mah and van Dyk, ${ }^{31}$ for example, predicted an increased risk of lung toxicity when homogeneous media was assumed for dose prescription (without a corresponding change in the prescription dose), whereas Chang et al. ${ }^{37}$ suggested that the homogeneous and heterogeneous dosimetric parameters are highly correlated, and therefore the pulmonary toxicity data from the existing literature are valid for both homogeneous and heterogeneous plans. The $9 \%$ average increase in MLD for the $6 \mathrm{MV}$ beam from our study is in very good agreement with the data from the first study mentioned above, but differs from the $4 \%$ average MLD increase reported in the second study. As we have found that the average change in MLD is not affected by the plan conformality, we can only speculate that the discrepancy between our study and the study by Chang et al. $^{37}$ is due to the patient selection and possibly the dose calculation algorithm used to correct for heterogeneities.

\section{B. Breathing-induced motion and deformation effects}

The minimal changes in CTV EUD and isocenter doses in face of motion are a consequence of an appropriate target design (ITV based on the union of exhale and inhale GTVs in this study). In addition, the motion-induced effects affect the penumbral regions, where high dose gradients exist over the range of motion, and thus they are unlikely to alter isocenter doses when the isocenter is located inside the target. Small EUD increases were found in several cases, and they were due to motion-induced hot spots that occurred at the edge of some beam segments that intersected the target.

The hot/cold spots seen in the dose difference maps from Fig. 7 are created in regions that move in/out of the radiation field during breathing. Of importance in the redistribution of the cumulative doses is how these hot and cold spots are located with respect to the normal lung tissue, as previously reported in a study involving intrahepatic lesions. ${ }^{53}$ Here one is presented with one of the two scenarios: (a) either the hot or the cold spot falls into the normal lung tissue, as illustrated in Figs. 7(b) and 7(d)-7(f); or (b) both the hot and the cold spots fall into the normal lung [in either one of the lungs, as in Fig. 7(c), or in both lungs, as in Fig. 7(a)]. As lungs are parallel organs, for which the response to radiation is correlated with the mean dose received, one can easily anticipate that when both the hot and the cold spots occur 
inside the lung they tend to offset each other, leaving the mean overall toxicity virtually unchanged, regardless of the motion amplitude. Patient 14, for example, underwent the largest tumor motion amplitude $(2.1 \mathrm{~cm})$, and yet, the change in normal lung toxicity was barely enough to allow for a fraction increase in the prescription dose in the conformal plan. By contrast, when either a hot or a cold motion induced spot occurred inside the lung, the overall lung toxicity increased/decreased compared to the corresponding static value. However, since the lungs are relative large-volume organs, the respective hot or cold spot would need to encompass a rather large portion on the normal tissue in order to have a measurable effect on the overall mean lung dose. This suggests that rather large motion amplitudes are required to create clinically significant changes in the mean lung dose. Small motion amplitudes are unlikely to lead to important changes, even when an uncompensated hot or cold spot exists [Fig. 7(e)].

In general, with respect to the cranio-caudal direction of motion, in this limited study the MLD is observed to: increase if the tumor is located near the diaphragm (moving inferiorly) or at the apex (moving superiorly), decrease for apical tumors moving inferiorly, and remain almost unchanged otherwise. It should also be noted that cranio-caudal motion components were usually larger and more likely to influence the redistribution of doses, as they generally occur perpendicular to the beam axis (for axial beams). Similarly, the lateral motion component can be expected to influence dose distributions in AP/PA beam settings; however, the motion amplitude along this direction is usually small.

An interesting finding of the motion component of this study is the overall decrease (albeit by a small amount) in the cumulative dose distribution MLD, when the entire population of patients was considered. Short of those cases where an uncompensated hot spot existed, the reduction of lung dose indicates, in fact, that lungs do not expand isotropically: the lower regions tend to undergo more motion and deformation relative to the upper regions. The overall result is that the MLD change is more driven by the cold spots thereby resulting in a slight decrease in the MLD.

The motion effects appear to be somewhat more pronounced for the higher beam energy, due to wider penumbra, especially inside the low-density lung tissue. However, the differences are rather small.

\section{Heterogeneity versus breathing-induced motion and deformation effects}

Not accounting for motion/deformation does not introduce significant errors in the target coverage assessment, a natural consequence of an appropriate planning margin. The margin expansion, however, cannot (and is not intended to) protect against the heterogeneity effects.

For the normal lung tissue, the heterogeneity effects alone are not sufficient to accurately evaluate doses when uncompensated hot or cold spots are created inside the lung as a result of large tumor excursions.
The magnitude of the dosimetric changes induced by motion at a point in space should not be associated with its influence on treatment metrics, as demonstrated by the example from Fig. 8 (Patient 14). There are two main causes for discrepancies between the magnitudes of the point dose changes and how these dosimetric changes affect lung NTCPs and MLDs. First, the motion-induced changes occur in smaller regions around beam edges. In contrast, heterogeneity effects are present everywhere inside the radiation field, and therefore, they will have a greater weight in the overall lung dose change. Second, in those cases where the hot and the cold spots occur inside the lung, their contributions offset each other, at least in part, thus decreasing their combined contribution to the overall lung dose.

The discrepancies between the heterogeneity and motioninduced effects on normal lung tend to be smaller at higher beam energies, as suggested by the correction factors derived from the linear fits illustrated in Fig. 6. This is a consequence of decreased heterogeneity effects, rather than a significant change in the magnitude of motion-induced effects with changing beam energy.

In this study, the heterogeneity effects were assessed only for the exhale dataset. Due to the reduced lung density during inhalation, the exhale dose usually overestimates the dose received by the lung tissue during any other phase of the breathing cycle, with the largest discrepancy occurring at inhale. The same applies to tumors (larger than coin size), for reasons related to decreased attenuation, as discussed previously. However, although there is a range of lung densities between inhalation and exhalation $\left(0.16\right.$ to $\left.0.35 \mathrm{~g} / \mathrm{cm}^{3}\right),{ }^{54}$ these values are different from the unit density assumed in homogeneous calculation in the first place. Therefore, the relative importance of the heterogeneity effects vs. motion effects is not expected to change significantly when inhale geometries are considered.

For the assessment of the motion/deformation effects, only the extreme patient geometries were included, based on a previous study ${ }^{47}$ indicating that cumulative doses can be estimated within $2 \%-3 \%$ accuracy when only the exhale and the inhale datasets are used. As such, the relative effects of motion and heterogeneity are expected to be quite robust, regardless of the number of breathing phases used for the cumulative dose reconstruction. In addition, the assumptions made in this study regarding the shape and the reproducibility of the breathing pattern are also expected to have minor influence on the relative comparison between heterogeneity and motion induced effects, as the effect of breathing variability on the cumulative dose is small, unless systematic deviations in the respiratory pattern during radiation delivery from the (assumed) planning model occur. ${ }^{47,50}$

The results of this study suggest that although large point dose changes are induced by both the lack of heterogeneity and motion/deformation corrections, tissue heterogeneity effects are likely to have a larger significance in terms of predicted clinical response parameters for tumor and normal lung (given a properly designed ITV). This may indicate the 
area of greater impact for investigations into dose calculations for lung tumor irradiations and quality assurance of treatment planning systems.

\section{ACKNOWLEDGMENTS}

This work was supported by Grants No. R01 CA106770 and No.P01 CA59872 from the National Cancer Institute.

${ }^{1}$ M. V. Graham et al., "Preliminary results of a prospective trial using three-dimensional radiotherapy for lung cancer," Int. J. Radiat. Oncol., Biol., Phys. 33, 993-1000 (1995).

${ }^{2}$ G. S. Sibley et al., "Radiotherapy alone for medically inoperable Stage I non-small-cell lung cancer The Duke experience," Int. J. Radiat. Oncol., Biol., Phys. 40, 149-154 (1998).

${ }^{3}$ J. D. Cox et al., "A randomized phase I/II trial of hyperfractionated radiation therapy with total doses of 60.0 Gy to 79.2 Gy: Possible survival benefit with $>$ or equal to 69.6 Gy in favorable patients with Radiation Therapy Oncology Group stage III non-small-cell lung carcinoma: Report of Radiation Therapy Oncology Group 83-11," J. Clin. Oncol. 8, 15431555 (1990).

${ }^{4}$ R. O. Dillman et al., "Improved survival in stage III non small cell lung cancer: Seven-year follow-up of Cancer and Leukemia Group B (CALGB) 8433 trial," J. Natl. Cancer Inst. 88, 1210-1215 (1996).

${ }^{5}$ W. T. Sause et al., "Radiation Therapy Oncology Group (RTOG) 88-08 and Eastern Cooperative Oncology Group (ECOG) 4588 Preliminary results of a phase III trial in regionally advanced, unresectable nonsmallcell lung cancer," J. Natl. Cancer Inst. 87, 198-205 (1995).

${ }^{6}$ C. A. Perez et al., "Patterns of tumor recurrence after definitive irradiation for inoperable non-oat cell carcinoma of the lung," Int. J. Radiat. Oncol., Biol., Phys. 6, 987-994 (1980).

${ }^{7}$ C. A. Perez et al., "Impact of tumor control on survival in carcinoma of the lung treated with irradiation," Int. J. Radiat. Oncol., Biol., Phys. 12, 539-547 (1986).

${ }^{8} \mathrm{~S}$. Vijayakumar et al., "Optimization of radical radiotherapy with beam's eye view techniques for non-small cell lung cancer," Int. J. Radiat. Oncol., Biol., Phys. 21, 779-788 (1991).

${ }^{9}$ F.-M. Kong et al., "High-dose radiation improved local tumor control and overall survival in patients with inoperable/unresectable non-small-cell lung cancer: Long-term results of a radiation dose escalation study," Int. J. Radiat. Oncol., Biol., Phys. 63, 324-333 (2005).

${ }^{10}$ J. M. Robertson et al., "Dose escalation for non-small-cell lung cancer using conformal radiation therapy,” Int. J. Radiat. Oncol., Biol., Phys. 37, 1079-1085 (1997).

${ }^{11}$ J. G. Armstrong et al., "Strategy for dose escalation using 3-dimensional conformal radiation therapy for lung cancer," Ann. Oncol. 6, 693-697 (1995).

${ }^{12}$ K. E. Rosenzweig et al., "Results of a Phase I dose escalation study in the treatment of inoperable non-small-cell lung cancer," Int. J. Radiat. Oncol., Biol., Phys., Suppl. 57, S417-S418 (2003).

${ }^{13}$ J. G. Rosenman et al., "High-dose conformal radiotherapy for treatment of Stage IIIA/IIIB non-small-cell lung cancer Technical issues and results of a Phase I/II trial," Int. J. Radiat. Oncol., Biol., Phys. 54, 348-356 (2002).

${ }^{14}$ J. S. A. Belderbos J. V. Lebesque, and I. Barillot, "Normal tissue complication probabilities for irradiation of NSCLC patients with and without elective nodal irradiation," Lung Cancer 18(Suppl.), 126 (1997).

${ }^{15} \mathrm{M}$. A. Socinski et al., "Induction and concurrent chemotherapy with highdose thoracic conformal radiation therapy in unresectable stage IIIA and IIIB non-small-cell lung cancer. A dose-escalation phase I trial," J. Clin. Oncol. 22, 4341-4350 (2004).

${ }^{16}$ R. K. Ten Haken et al., "Use of Veff and iso-NTCP in the implementation of dose escalation protocols," Int. J. Radiat. Oncol., Biol., Phys. 27, 689-695 (1993).

${ }^{17}$ J. A. Hayman et al., "Dose escalation in non-small-cell lung cancer using three-dimensional conformal radiation therapy. Update of a phase I trial," J. Clin. Oncol. 19, 127-136 (2001).

${ }^{18} \mathrm{~S}$. Narayan et al., "Results following treatment to doses of 92.4 or 102.9 Gy on a phase I dose escalation study for non-small cell lung cancer," Lung Cancer 44, 79-88 (2004).

${ }^{19}$ M. J. Berger and S. M. Seltzer, "ETRAN, Monte Carlo code system for electron and photon transport through extended media," ROSC computer package CCC-107, Oak Ridge National Laboratory, Oak Ridge, TN, 1973.

${ }^{20}$ F. Salvat et al., "PENELOPE, an algorithm and computer code for Monte Carlo simulation of electron-photon shower," Ciemant, Report No. 799, 1992.

${ }^{21}$ C. M. Ma et al., "MCDOSE - a Monte Carlo dose calculation tool for radiation therapy treatment planning," Proceedings of the XIII International Conference on the Use of Computer in Radiation Therapy (Springer-Verlag, Heidelberg, 2000), pp. 123-125.

${ }^{22}$ M. Goosens, S. Giani, and S. Ravndal, "GEANT: detector description and simulation tool," Technical Report CERN Program library, long writeup W5013 CERN, Geneva, Switzerland, 1993.

${ }^{23}$ P. J. Keall and P. W. Hoban, "Super-Monte Carlo: A 3D electron beam dose calculation algorithm," Med. Phys. 23, 2023-2034 (1996).

${ }^{24}$ M. Fippel, "Fast Monte dose calculation for photon beams based on the VMC electron algorithm," Med. Phys. 26, 1466-1475 (1999).

${ }^{25}$ J. Deng et al., "Photon beam characterization and modeling for Monte Carlo treatment planning," Phys. Med. Biol. 45, 411-427 (2000).

${ }^{26}$ J. Sempau, S. J. Wilderman, and A. F. Bielajew, "DPM, a fast, accurate Monte Carlo code optimized for photon and electron radiotherapy treatment planning dose calculations," Phys. Med. Biol. 45, 2263-2291 (2000).

${ }^{27}$ I. J. Chetty, J. J. DeMarco, and T. D. Solberg, "A virtual source model for Monte Carlo modeling of arbitrary intensity distributions," Med. Phys. 27, 166-172 (2000).

${ }^{28} \mathrm{M}$. K. Fix et al., "A multiple source model for $6 \mathrm{MV}$ photon beam dose calculations," Phys. Med. Biol. 46, 1407-1428 (2001).

${ }^{29}$ "VMC++, electron and photon Monte Carlo calculations optimized for Radiation Treatment Planning," Advanced Monte Carlo for Radiation Physics, Particle Transport Simulation and Applications: Proceedings of the Monte Carlo Meeting, Lisbon, edited by A. Kling, F. Barao, M. Nakagawa, L. Tavora, and P. Vaz (Springer, Berlin, 2001), pp. 229-236.

${ }^{30}$ N. Papanikolaou et al., AAPM Report No. 85: "Tissue inhomogeneity corrections for megavoltage photon beams," Report of Task Group No. 65 of the Radiation Therapy Committee of the American Association of Physicists in Medicine, 2004.

${ }^{31} \mathrm{~K}$. Mah and J. Van Dyk, "On the impact of tissue inhomogeneity corrections in clinical thoracic radiation therapy,” Int. J. Radiat. Oncol., Biol., Phys. 21, 1257-1267 (1991).

${ }^{32}$ C. G. Orton et al., "Study of lung density corrections in a clinical trial (RTOG 88-08)," Int. J. Radiat. Oncol., Biol., Phys. 41, 787-794 (1998). ${ }^{33} \mathrm{~A}$. Van't Riet et al., "Implications of lung corrections for dose specification in radiotherapy," Int. J. Radiat. Oncol., Biol., Phys. 11, 621-625 (1985).

${ }^{34}$ W. G. McKenna et al., "Is correction for lung density in radiotherapy treatment planning necessary?," Int. J. Radiat. Oncol., Biol., Phys. 13, 273-278 (1987).

${ }^{35} \mathrm{C}$. G. Orton et al., "Lung corrections in photon beam treatment planning: are we ready?,” Int. J. Radiat. Oncol., Biol., Phys. 10, 2191-2199 (1984). ${ }^{36}$ N. Papanikolaou, E. E. Klein, and W. R. Hendee, "Heterogeneity corrections should be used in treatment planning for lung cancer?," Med. Phys. 27, 702-1704 (2004).

${ }^{37} \mathrm{D}$. T. Chang et al., "The impact of heterogeneity correction on dosimetric parameters that predict for radiation pneumonitis," Int. J. Radiat. Oncol., Biol., Phys. 65, 125-131 (2006).

${ }^{38} \mathrm{P}$. J. Keall et al., "Time - The fourth dimension in radiotherapy, ASTRO panel presentation,” Int. J. Radiat. Oncol., Biol., Phys., Suppl. 57, S8-S9 (2003).

${ }^{39}$ A. E. Lujan et al., "A method for incorporating organ motion due to breathing into 3D dose calculations," Med. Phys. 26, 715-720 (1999).

${ }^{40}$ I. J. Chetty et al., "Photon beam relative dose validation of the DPM Monte Carlo code in lung-equivalent media," Med. Phys. 30, 563-573 (2003).

${ }^{41}$ I. J. Chetty et al., "Reporting and analyzing statistical uncertainties in Monte Carlo-based treatment planning," Int. J. Radiat. Oncol., Biol., Phys. 65, 1249-1259 (2006).

${ }^{42}$ M. Rosu et al., "Dose reconstruction in deforming lung anatomy: dose grid size effects and clinical implications," Med. Phys. 32, 2487-2495 (2005).

${ }^{43}$ G. J. Kutcher and C. Burman, "Calculation of complication probability factors for non-uniform normal tissue irradiation: the effective volume method,” Int. J. Radiat. Oncol., Biol., Phys. 16, 1623-1630 (1989). 
${ }^{44}$ J. Lyman, "Complication probability as assessed from dose volume histograms," Radiat. Res. 104, S13-S19 (1985).

${ }^{45}$ Y. Seppenwoolde et al., "Comparing different NTCP models that predict the incidence of radiation pneumonitis. Normal tissue complication probability,” Int. J. Radiat. Oncol., Biol., Phys. 55, 724-735 (2003).

${ }^{46}$ M. L. Kessler and M. Roberson, "Image registration and data fusion for radiotherapy treatment planning," in New Technologies in Radiation, edited by W. Schlegel, T. Bortfeld, and A. L. Grosu (Springer-Verlag, Berlin, 2005), pp. 41-52.

${ }^{47}$ M. Rosu et al., "How extensive of a 4D dataset is needed to estimate cumulative dose distribution plan evaluation metrics in conformal lung therapy?," Med. Phys. 34, 233-245 (2007).

${ }^{48}$ A. Niemierko, "A generalized concept of equivalent uniform dose (EUD),” Med. Phys. 26, 1100 (1999).

${ }^{49}$ R. K. Ten Haken et al., "Potential benefits of eliminating planning target volume expansions for patient breathing in the treatment of liver tumors,"
Int. J. Radiat. Oncol., Biol., Phys. 38, 613-617 (1997).

${ }^{50}$ A. E. Lujan, J. M. Balter, and R. K. Ten Haken, "A method of incorporating organ motion due to breathing into $3 \mathrm{D}$ dose calculations in the liver: Sensitivity to variations in motion," Med. Phys. 30, 2643-2649 (2003).

${ }^{51}$ T. R. Mackie, J. W. Scrimger, and J. J. Battista, “A convolution method of calculating dose for 15-MV x-rays,” Med. Phys. 12, 188-196 (1985).

${ }^{52} \mathrm{E}$. Yorke et al., "Dosimetric considerations in radiation therapy of coin lesions of the lung," Int. J. Radiat. Oncol., Biol., Phys. 34, 481-487 (1996).

${ }^{53}$ M. Rosu et al., "Alterations in normal liver doses due to organ motion," Int. J. Radiat. Oncol., Biol., Phys. 57, 1472-1479 (2003).

${ }^{54}$ J. Van Dyk, T. J. Keane, and W. D. Rider, "Lung density measured by computerized tomography: implications for radiotherapy,” Int. J. Radiat. Oncol., Biol., Phys. 8, 1363-1372 (1982). 